\title{
Combined CT guided tru-cut biopsy and imprint cytology in the diagnosis of bone tumors and tumor like lesions
}

\author{
Moothiringode Chitrabhanu Savithri', Kanjirakkad Kavitha', Dominic Puthoor ${ }^{2}$ \\ Associate Professor, ${ }^{1}$ Departments of Pathology and ${ }^{2}$ Orthopedics, Amala Institute of Medical Sciences, Amalanagar,
}

Thrissur, Kerala, India

\section{A B S T R A C T}

Background: Bone lesions are often histologically heterogenous. The advantage of trucut biopsies in this context is that they provide a safe and accurate biopsy technique that can be performed in less specialized centers. In this series we found combination of biopsy and cytology helpful in diagnosing bone lesions. However clinical picture and radiologic features are vital in arriving at a final diagnosis. Aims and Objectives: 1) To assess the diagnostic accuracy of combined CT guided tru-cut biopsy and imprint cytology in the diagnosis of bone tumors and tumor like lesions. 2) To analyze the spectrum of different bone lesions. Materials and Methods: 108 consecutive cases of bone lesions for which CT guided tru-cut biopsy and imprint cytology were done between June 2010 and april 2012 were retrospectively studied. Both smears and biopsies were categorized into five groups.Correlation between biopsy and cytology were also evaluated. Results: Total number of cases were 108 of which 8 cases were inconclusive. In 100 cases a useful opinion could be given. There were 36 cases of primary bone tumors, 13 cases of plasma cell neoplasms, 42 metastatic tumors, 9 non neoplastic lesions and 8 inconclusive cases. Conclusion: Preliminary diagnosis of bone lesions before definitive therapy helps to avoid unnecessary surgical procedures. Tru cut biopsies supplemented by imprint cytology can provide definitive diagnosis in majority of cases.

Access this article online

\section{Website:}

http://nepjol.info/index.php/AJMS

DOI: 10.3126/ajms.v7i6.13310

E-ISSN: 2091-0576

P-ISSN: 2467-9100

Key words: Bone lesions, Imprint cytology, Tru-cut biopsy

\section{INTRODUCTION}

Tru-cut biopsies are popular and relatively easier method for getting a primary diagnosis before deciding on specific therapy in many sites especially in breast lesions. Bone lesions are often histologically more heterogenous. Imprint cytology smears made along with tru-cut biopsies in bone lesions can be helpful in the final diagnosis. In this series we found combination of biopsy and cytology helpful in diagnosing bone lesions. However clinical picture and radiologic features are vital in arriving at a final diagnosis.
Aims

1) To assess the diagnostic accuracy of combined CT guided tru-cut biopsy and imprint cytology in the diagnosis of bone tumors and tumor like lesions.

2) To assess the spectrum of different bone lesions.

\section{MATERIALS AND METHODS}

One hundred -eight consecutive cases of bone lesions for which CT guided tru-cut biopsy and imprint cytology were done between June 2010 and April 2012 were retrospectively studied. Imprints were made immediately 
by the surgeon by rolling the tissue on glass slides. At least one slide was air dried for MGG staining and the rest were fixed in isopropyl alcohol for papanicolaou staining.

Biopsies were formalin fixed, routinely processed and sections stained by Hematoxylin Eosin stain.

Both smears and biopsies were categorized into

A) Primary bone tumors

B) Plasma cell neoplasms

C) Metastatic tumors

D) Non tumor lesions and

E) Inconclusive.

Correlation between biopsy and cytology were also evaluated.

\section{RESULTS}

Total number of cases were 108 of which 8 cases were inconclusive. In 100 cases-92.5\%- a useful opinion could be given (Table 1).

Category A comprised $33.3 \%$ of lesions. They included GCT, OGS, chondrosarcoma, Ewing sarcoma, Lymphoma, chondroblastoma and Langerhan cell histiocytosis $(\mathrm{LCH})$ (Table 2).

In 2 cases of osteogenic sarcoma the cytology diagnosis was limited to positive for malignant cells. Most cases of OGS showed cellular smears with malignant spindle cells but identification of osteiod was tough. $\mathrm{X}$ ray correlation helped in giving a final diagnosis in these cases. Two cases were parosteal OGS in which nuclear features of malignancy were more clear with cytology.

\section{Table 1: Lesion categories}

\begin{tabular}{llc} 
Category & Lesions & No. of cases \\
\hline A & Primary tumors & 36 \\
B & Plasma cell neoplasms & 13 \\
C & Metastasis & 42 \\
D & Non tumor lesions & 09 \\
E & Inconclusive & 08 \\
\hline
\end{tabular}

\begin{tabular}{lccc}
\multicolumn{4}{l}{ Table 2: Primary bone tumors } \\
\hline Tumor type & $\begin{array}{c}\text { No. of } \\
\text { cases }\end{array}$ & $\begin{array}{c}\text { Cytology } \\
\text { diagnosis }\end{array}$ & $\begin{array}{c}\text { Biopsy } \\
\text { diagnosis }\end{array}$ \\
\hline GCT & 11 & 11 & 11 \\
OGS & 11 & 09 & 11 \\
Chondrosarcoma & 03 & 02 & 01 \\
Ewing sarcoma & 04 & 04 & 04 \\
Lymphoma & 03 & 03 & 03 \\
Chondroblastoma & 02 & 01 & 02 \\
LCH & 02 & 02 & 02 \\
\hline
\end{tabular}

There were 3 cases of chondrosarcoma. In one case cytology was suggestive of a cartilaginous lesion and in the second one it was suggestive of chondrosarcoma. The third case was inconclusive cytologically but biopsy was diagnostic.

All cases of lymphomas were called malignant round cell neoplasms by cytology. Biopsy was conclusive with immunohistochemical support. Discordance between cytology and biopsy were noted in one case of chondroblastoma which was cytologically diagnosed as GCT.

Plasma cell neoplasms (Category B) formed 12\% where all 13 cases could be diagnosed cytologically and histologically.

Metastatic carcinoma (Category C) comprised 42 cases $(38 \%)$ and all were adenocarcinomas. This was the most frequent group. 39 cases showed correlation between cytologic and histologic diagnosis. One case did not have adequate material in the cytology smears. Two cases of metastatic carcinoma interestingly could be diagnosed cytologically in spite of low cellularity but definite opinion was difficult in biopsy sections.

Category $\mathrm{D}$ included aneurismal bone cyst (ABC), simple bone cyst (SBC), fibrous dysplasia (FD) and one case of gout and formed $8.3 \%$ of total (Table 3 ).

There were 4 cases of $A B C$ and 3 cases were suggestive in imprint cytology. One case showed only blood. Both cases of SBC were indicative of a benign cystic lesion with cyst macrophages. Biopsy was inadequate in one case.

Both cases of FD showed a few benign spindle cells in the smears. One case was diagnosed FD in trucut biopsy and the other could be labeled as benign fibro osseous lesion in biopsy.

The one case of gout showed only amorphous material in cytology smears but showed features of gout in histology.

Only 8 cases were inconclusive where no diagnosis was possible by cytology and biopsy.

\begin{tabular}{lccc}
\multicolumn{4}{l}{ Table 3: Non tumor lesions } \\
\hline Lesion & $\begin{array}{c}\text { No. of } \\
\text { cases }\end{array}$ & $\begin{array}{c}\text { Cytology } \\
\text { diagnosis }\end{array}$ & Biopsy diagnosis \\
\hline ABC & 4 & 3 & 4 \\
SBC & 2 & 2 & 1 \\
FD & 2 & Inconclusive & 1 \\
Gout & 1 & Inconclusive & 1 \\
\hline
\end{tabular}




\section{DISCUSSION}

Bone tumors comprise a diverse spectrum of morphologic and behavioral entities. They may be benign, malignant or potentially malignant. There are many tumor like lesions also in the bone. Correct diagnosis is important in choosing therapeutic modalities.

Preoperative diagnosis depends on good clinical examination and radiologic assessment. However tissue diagnosis is essential before starting definitive treatment especially in cases where radical surgery or chemotherapy is indicated.

Open biopsy is a common method the advantage being the availability of ample material for evaluation. Many consider this as the gold standard but there can be significant contamination of the surrounding tissue by tumor cells.this had little significance in the past because most malignancies were treated by amputation. Now the scenario has changed with advances in surgical techniques, limb salvage surgeries, and modern chemotherapy regimens.

The advantage of tru-cut biopsies in this context is that they provide a safe and accurate biopsy technique that can be performed in less specialized centers. Joshi A et al found the sensitivity and specificity of Tru-cut biopsy of palpable bone lesions with soft tissue extension in their series was $92.85 \%$ and $100 \%$, respectively, with positive predictive value of $100 \%$ and negative predictive value of $57.14 \% .{ }^{1}$ However guided biopsies are becoming popular as they can hit the lesion more accurately in many cases. Ultrasound guided tru-cut biopsies were done by Saifiddin et al in lesions with relatively large extraosseous component. For pure intramedullary lesions with minimal extraosseous spread they preferred fluoroscopy guidance and more inaccessible lesions were biopsied under CT guidance. ${ }^{2}$

Imprint smears are made in bone marrow biopsies almost routinely. Trephine biopsies can provide information about the structure of relatively large pieces of marrow. Individual cell morphology may be studied by making imprints from the biopsy core. ${ }^{3}$ This concept has been tried in many areas with success. In lung tumors this has been found to be reliable and can be used to provide a rapid, preliminary diagnosis of lung cancer. ${ }^{4}$ There are also studies on imprint cytology of prostate core biopsies. In one such series cytology identified malignant cells more effectively. ${ }^{5}$
Ayhan et al combined tru-cut biopsy with Fine Needle Aspiration Biopsy with high consistency rate in jaw lesions. ${ }^{6}$ A study on Fine needle aspiration cytology of bone lesions from Cairo University concluded that FNAC could differentiate various giant cell rich lesions and round cell malignancies like Ewing sarcoma, myeloma and. ${ }^{7} \mathrm{We}$ found combining imprint cytology with tru-cut biopsy improved understanding of cytologic features. In the relatively bland appearing parosteal OGS nuclear atypia was more evident in cytology thus precluding a diagnosis of benign lesion. The nuclear grooves of chondroblastoma, typical histiocytes of LCH and cells plasmacytoma were all well demonstrated in cytology. It was in the cases of benign fibro osseous lesions that cytology faltered. But rapid diagnosis was possible in most malignancies and other primary bone tumors.

\section{CONCLUSION}

Preliminary diagnosis of bone lesions before definitive therapy helps to avoid unnecessary surgical procedures. Tru cut biopsies supplemented by imprint cytology can provide definitive diagnosis in majority of cases.

\section{REFERENCES}

1. Joshi A, Magar SR, Chand P, Panth R and Chhetri BRK. Tru-cut biopsy as the initial method of tissue diagnosis in bone tumors with soft tissue extension. Indian J Orthop 2013;47:195-199.

2. Saifuddin A, Mitchell R, Burnett SJD, Sandison A and Pringle JAS. Ultrasound guided needle biopsy of primary bone tumors. J Bone Joint Surg [Br] 2000;82-B:50-54.

3. Metikurke $\mathrm{SH}$, Rashmi $\mathrm{K}$ and Bhavika R. Correlation of bone marrow aspirate, biopsies and touch imprint findings in pancytopenia. J Hematol 2003;2:8-13.

4. Paulose RR, Shee CD, Abdelhadi IA and Khan MK. Accuracy of touch imprint cytology in diagnosing lung cancer. Cytopathology 2004; 15:109-112.

5. Aytac B, Atalay FO, Vuruskan H and Filiz G. Touch imprint cytology of prostate core needle biopsy specimen: A useful method for immediate reporting of prostate cancer. J Cytol 2012; 29:173-176.

6. Ayhan NK, Keskin C, Olgac V, Dedeoglu K, Yaltiric M, Erseven G, et al. Tru-cut and fine needle aspiration biopsy diagnosis of lesions of jaws. Turk J Med Sci 2003; 33:249-253.

7. Aly AM, Shaaban HM and Abou-Sinna. Accuracy of fine needle aspiration cytology in the diagnosis of bone lesions with radiological assistance: Experience from the National Cancer Institute, Cairo University, Egypt. Egypt J Radiol Nucl Med 2014; 45:127-135.

\footnotetext{
Authors Contribution:

MCS - Concept and design of the study, reviewed the literature, manuscript preparation and critical revision of the manuscript; KPK - Concept, collected data and review of literature, statistically analyzed and helped in preparing first draft of manuscript; DP - Conceptualized study, literature search, and critical revision of the manuscript.

Source of Support: Nil, Conflict of Interest: None declared.
} 